\title{
A Novel Nonmechanical Finger Rehabilitation System Based on Magnetic Force Control
}

\author{
In-Chul Baek ${ }^{1}$, Min Su Kim², and Sung Hoon Kim ${ }^{1 *}$ \\ ${ }^{I}$ Department of Electronics Convergence Engineering, Wonkwang University, 460 Iksandae-ro, Iksan, \\ Jeonbuk 54538, Republic of Korea \\ ${ }^{2}$ Department of Rehabilitation Medicine, School of Medicine Wonkwang University, 859 Muwang-Ro, Iksan, \\ Jeonbuk 54538, Republic of Korea
}

(Received 7 February 2017, Received in final form 4 March 2017, Accepted 6 March 2017)

\begin{abstract}
This paper presents a new nonmechanical rehabilitation system driven by magnetic force. Typically, finger rehabilitation mechanisms are complex mechanical systems. The proposed method allows wireless operation, a simple configuration, and easy installation on the hand for active actuation by magnetic force. The system consists of a driving coil, driving magnets (M1), and auxiliary magnets (M2 and M3), respectively, at the finger, palm, and the center of coil. The magnets and the driving coil produce three magnetic forces for an active motions of the finger. During active actuations, magnetic attractive forces between M1 and M2 or between M1 and M3 enhance the flexion/extension motions. The proposed system simply improves the extension motion of the finger using a magnetic system. In this system, the maximum force and angular variation of the extension motion were $0.438 \mathrm{~N}$ and $49^{\circ}$, respectively. We analyzed the magnetic interaction in the system and verified finger's active actuation.
\end{abstract}

Keywords : magnetic force, finger rehabilitation, electromagnetic system, active finger actuation

\section{Introduction}

Stroke is a serious problem that is rapidly increasing throughout the world. One major symptom of stroke is hand injury. Even when there is significant improvement in the patient's condition, rehabilitation training is most important for post-stroke care. For hand rehabilitation training, various assistive devices have been reported [13]. The majority of these assistive devices are mechanical systems, such as robot-assisted therapy [4,5]. Most mechanical systems for hand rehabilitation have drawbacks, such as complex configurations, the difficulty of installing them on a finger, and their large volume. They are divided into hard types and soft types, such as roboticgloves $[6,7]$. In general, the hard type devices consist of electrical motors, wires, frames, and various mechanical components. However, the hard type devices provide active actuation (external force) using motors (electrical or pneumatic actuators) $[8,9]$. Soft type devices (flexible

CThe Korean Magnetics Society. All rights reserved.

*Corresponding author: Tel: +82-63-850-6739

Fax:+82-63-850-6739, e-mail: kshoon@wku.ac.kr gloves) have a relatively simple configuration, they are difficult to install on the fingers. These the devices are also driven by the stroke patient's own hand force, using a virtual reality system or computer graph [10]. Typically, the degree of freedom (DOF) of robotic glove systems is limited, which also then limits the flexion and extension motions. To increase the DOF of finger movement, the number of actuators must increase, resulting in the system becoming more and more complex and inconvenient.

To avoid these issues, we proposed a novel method based on the magnetic actuation of a hand rehabilitation system using an electromagnetic system with permanent magnets. The system mainly utilizes magnetic attractive and repulsive forces between the driving coil and the permanent magnet and between the permanent magnets themselves. The magnetic force becomes an energy source for wireless control. The wireless magnetic controls have been used for magnetic microrobots [11-13]. The proposed method, which has higher DOFs of motion than those of robotic gloves due to magnetic force directions, does not require mechanical components (as does a kinetic system). The developed system consists of a driving coil, permanent magnets on a finger and the palm, and permanent 
magnets inside coils. The magnetic force causes active flexion/extension motions or twist motions of the finger according to changes in the force direction. Various experimental analyses were performed to verify active finger rehabilitation training.

\section{Description of Control Method and System Configuration}

The proposed system is driven by magnetic force as a wireless operation. Figure 1(a) shows the principle of magnetic actuation between magnets or between a magnet and an electromagnet. Magnetic force is divided into an attractive force and a repulsive force. The developed system utilized cylindrical permanent magnets (M1, M2, and M3) and a circular coil, as shown in Fig. 1(b). The permanent magnets M1, M2, and M3 are installed, respectively, on a finger, on the palm, and inside the coil. M1 and M3 are equivalent magnets; their diameter is 20 $\mathrm{mm}$, and their thickness is $6 \mathrm{~mm}$. M2 has a diameter of $10 \mathrm{~mm}$ and a thickness of $3 \mathrm{~mm}$. Table 1 shows the magnetic properties of the installed magnets. $\mathrm{Br}$ is the residual magnetic flux density. Bs is the surface magnetic flux density. $(\mathrm{BH})_{\max }$ is the maximum energy product. $\mathrm{Hcb}$ is the coercive force. The inner and outer diameters of the driving coil are $10 \mathrm{~cm}$ and $15.3 \mathrm{~cm}$, respectively. The number of turns of the coil is 250 . Figure 1(c) shows the coordinate system of the finger and the magnetic
Table 1. Magnetic properties of the magnets.

\begin{tabular}{ccc}
\hline \hline & M1=M3 (N52) & M2 (N35) \\
\hline $\mathrm{Br}[\mathrm{mT}]$ & $1430-1480$ & $1170-1220$ \\
$\mathrm{Bs}[\mathrm{mT}]$ & 313 & 287 \\
$(\mathrm{BH})_{\max }\left[\mathrm{kj} / \mathrm{m}^{3}\right]$ & $394-422$ & $263-287$ \\
$\mathrm{Hcb}[\mathrm{kA} / \mathrm{m}]$ & $\geq 796$ & $\geq 868$ \\
\hline
\end{tabular}

forces for flexion and extension motions of the finger. The initial posture of the finger is $55^{\circ}\left(\phi_{e}=\theta_{1}+\theta_{2}+\theta_{3}\right)$. In the system, the magnetic forces are divided into three. First, $\boldsymbol{F}_{\boldsymbol{M 1 , 2}}$ and $\boldsymbol{F}_{\boldsymbol{M I , 3}}$ are attractive forces between M1 and M2 and between M1 and M3, respectively. $\boldsymbol{F}_{\boldsymbol{M I}, \boldsymbol{E M}}$ is the magnetic force between M1 and the driving coil. $\boldsymbol{F}_{\boldsymbol{M 1 , 2}}$ and $\boldsymbol{F}_{\boldsymbol{M I , 3}}$ are always attractive force because of the fixed configuration of the magnets, whereas $\boldsymbol{F}_{\boldsymbol{M I}, E M}$ is either an attractive force or a repulsive force according to the direction of current at the driving coil.

For flexion motion at the reference posture, we applied a current signal from 0 to $10 \mathrm{~A}$. When this occurs, $\boldsymbol{F}_{M 1, E M}$ becomes a repulsive force, which causes a flexion motion. In addition, when M1 is closed to M2, $\boldsymbol{F}_{\boldsymbol{M I}, 2}$ becomes an attractive force, and $\boldsymbol{F}_{\boldsymbol{M I}, \boldsymbol{E M}}$ is diverted from a repulsive force to an attractive force. For extension motion from the reference posture, we applied a current from 0 to $-10 \mathrm{~A}$. Under these conditions, $\boldsymbol{F}_{\boldsymbol{M I}, \boldsymbol{E M}}$ first becomes an attractive force, and $\boldsymbol{F}_{\boldsymbol{M I}, 3}$ enhances the extension motion as an attractive force. At the maximum flexion and extension
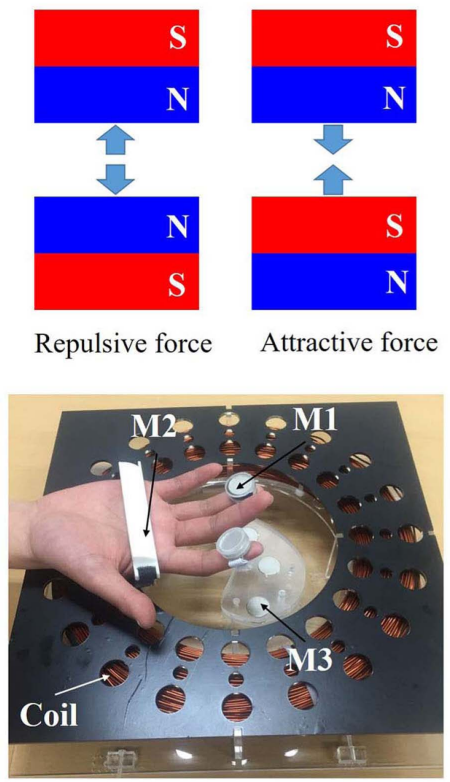
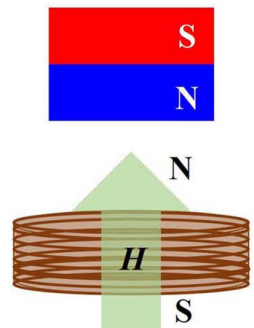

Repulsive force (a)

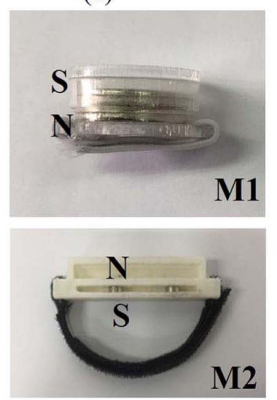

(b)
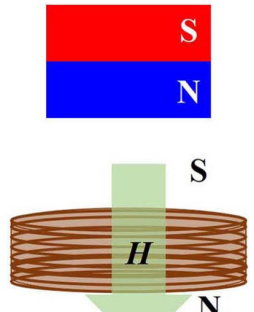

Attractive force

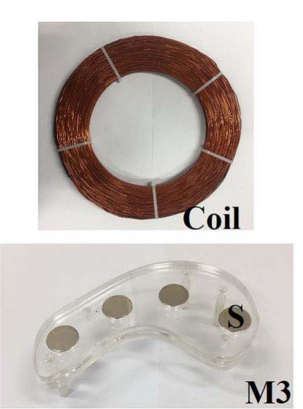

M3
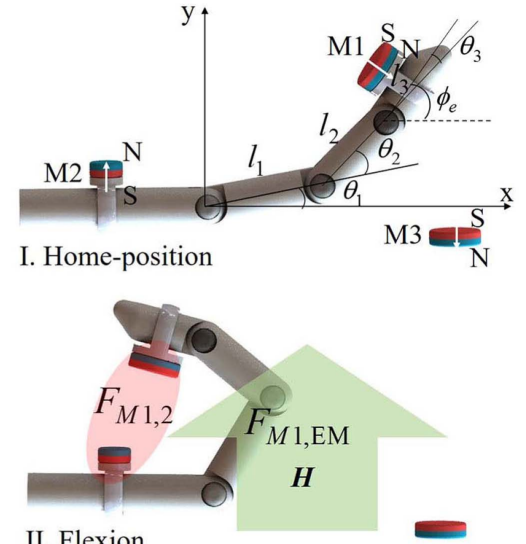

II. Flexion



(c)

Fig. 1. (Color online) (a) Principle of magnetic actuation in the proposed system, (b) system configuration, and (c) mechanism of extension and flexion motions of the finger by magnetic forces in the proposed configuration. 
motion, $\mathrm{M} 1$ and $\mathrm{M} 2$ have restricted air gap of $1.9 \mathrm{~cm}$, and $\mathrm{M} 1$ and M3 have a restricted air gap of $4.5 \mathrm{~cm}$, to maintain the force of the restoration of flexion and extension motion, because a larger attractive force cannot generate a repetitive exercise of the finger. $\boldsymbol{F}_{M 1, E M}$ causes weak flexion and extension motion, and $\boldsymbol{F}_{\boldsymbol{M} 1,2}$ and $\boldsymbol{F}_{\boldsymbol{M} \mathbf{1 , 3}}$ enhance the flexion and extension motion.

The general magnetic force between the cylindrical magnets is analyzed using the Gilbert model [14]. In this method, if the cylindrical magnets are far from each other, the magnetic interactive force is expressed as follows [15]:

$$
R^{4} \sum_{i j=0}^{1} \frac{(-1)^{i+j}}{\left(x+i t_{1}+j t_{2}\right)^{2}}\left[1-\frac{3}{2} \frac{r^{2}}{\left(x+i t_{1}+j t_{2}\right)^{2}}\right]
$$

where $M_{s}$ is the magnetization, $R$ is the magnets radius, $x$ is the distance between magnets, $t$ is the thickness of the magnet, and $\mathrm{r}$ is the lateral displacement, as shown in Fig. 2(a). If $t_{1}=t_{2}=t$ and $r=0$ then $\boldsymbol{F}$ can be rewritten as follows:

$$
F \approx-\frac{1}{4} \pi M_{s}^{2} R^{4}\left[\frac{1}{x^{2}}+\frac{1}{(x+2 t)^{2}}-\frac{2}{(x+t)^{2}}\right]
$$

When $t<<x$, Eq. (2) is reached at $F \approx 3 \pi \mu_{0} M_{s}^{2} R^{4} t^{2} / 2 x^{4}$. Eqs. (1) and (2) can be utilized at a linear distance. On the other hand, when there is no linear distance of the magnets, as shown in Fig. 2(b), their interactive force can be analyzed by magnetic dipole-dipole interaction. In the global coordinate system the interactive force can be expressed as follows $[14,16]$ :

$$
\begin{aligned}
& \boldsymbol{F}_{\boldsymbol{r}}(\boldsymbol{r}, \alpha, \beta)=-\frac{3 \mu_{0}}{4 \pi} \frac{\boldsymbol{m}_{1} \boldsymbol{m}_{2}}{\mathrm{r}^{4}} \\
& \quad[2 \cos (\delta-\alpha) \cos (\delta-\beta)-\sin (\delta-\alpha) \sin (\delta-\beta)] \\
& \boldsymbol{F}_{\delta}(\boldsymbol{r}, \alpha, \beta)=-\frac{3 \mu_{0}}{4 \pi} \frac{\boldsymbol{m}_{1} \boldsymbol{m}_{2}}{\mathrm{r}^{4}} \sin (2 \delta-\alpha-\beta)
\end{aligned}
$$

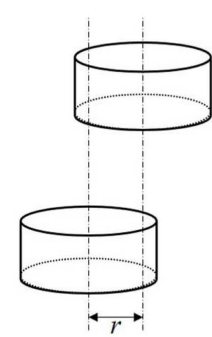

(a)



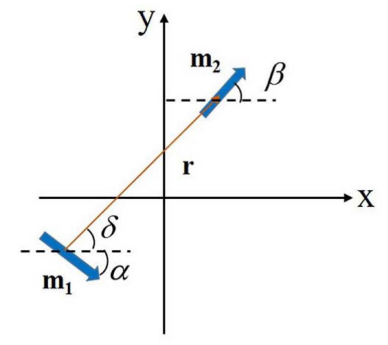

(b)
Fig. 2. (Color online) (a) Magnetic axial coupling for force analysis and (b) two separated dipoles. where $\mu_{0}$ denotes the permeability of free space. $\boldsymbol{m}_{\mathbf{1}}$ and $\boldsymbol{m}_{\mathbf{2}}$ are the dipole moment, and $\mathrm{r}$ is the distance vector between $\boldsymbol{m}_{1}$ and $\boldsymbol{m}_{2}$. In the proposed system, the position of the magnetic dipole moments $\left(\boldsymbol{m}_{\mathbf{1}}\right.$ and $\left.\boldsymbol{m}_{2}\right)$ can be observed by a kinetic analysis of the finger, as shown in Fig. 1(c). Magnets M2 and M3 and the driving coil have a fixed position, while M1 shows movement by an external magnetic field. The position of M1 is $P_{M 1}\left(x_{e}, y_{e}, \phi_{e}\right)$. A finger consists of three joints and links. In this case, the position of an end-effector (M1) is expressed as follows:

$$
\begin{aligned}
& x_{e}=l_{1} \cos \theta_{1}+l_{2} \sin \left(\theta_{1}+\theta_{2}\right)+l_{3} \cos \left(\theta_{1}+\theta_{2}+\theta_{3}\right) \\
& y_{e}=l_{1} \sin \theta_{1}+l_{2} \sin \left(\theta_{1}+\theta_{2}\right)+l_{3} \sin \left(\theta_{1}+\theta_{2}+\theta_{3}\right) \\
& \phi_{e}=\theta_{1}+\theta_{2}+\theta_{3}
\end{aligned}
$$

Where the length of links is $l_{1}, l_{2}$, and $l_{3}$, and $\theta_{1}, \theta_{2}$, and $\theta_{3}$ are the joint angles; and $x_{e}$ and $y_{e}$ are the $\mathrm{x}$ and $\mathrm{y}$ positions of the end effector. $\phi_{e}$ is the angle of the end effector. Thus, all magnetic forces in the system can be applied to Eqs. (1) to (4) for the flexion and extension motions of the finger.

\section{Experimental Analysis}

To verify the proposed mechanism, we conducted a performance evaluation through magnetic simulation, force analysis, and motion analysis using a video recording. In order to generate flexion and extension motion, we applied a current signal to the driving coil up to $\pm 10 \mathrm{~A}$. Figure 3(a) shows the simulation results of the driving coil at the applied current of $10 \mathrm{~A}$. The driving coil generated an average field of $11 \mathrm{kA} / \mathrm{m}$ and $7.2 \mathrm{kA} / \mathrm{m}$, at the heights of $4.5 \mathrm{~cm}$ and $8.5 \mathrm{~cm}$, respectively. Figure 3(b) shows the measured results according to changes in height and current. A height of $4.5 \mathrm{~cm}$ represented a maximum extension point of the forefinger in the system, and a height of $8.5 \mathrm{~cm}$ represented a maximum point during flexion motion. When we compared the magnetic field strength between the simulation and the measurements, the measured results were lower than those in the simulation at high current, because an increase in the temperature of the driving coil caused a decrease of field strength at high current. Therefore, the measured fields at $4.5 \mathrm{~cm}$ and $8.5 \mathrm{~cm}$ were averaged at $9.6 \mathrm{kA} / \mathrm{m}$ and 6.2 $\mathrm{kA} / \mathrm{m}$, respectively.

Figure 4 shows the trajectory of the forefinger and the magnetic field strength from the driving coil according to the trajectory. The $\mathrm{H}$ to $\mathrm{A}$ points are variations in the extension motion, and the $\mathrm{H}$ to $\mathrm{O}$ points are variations in the flexion motion. Because point $\mathrm{H}$ is the reference, the magnetic field strength of point $\mathrm{H}$ is zero. To start the 


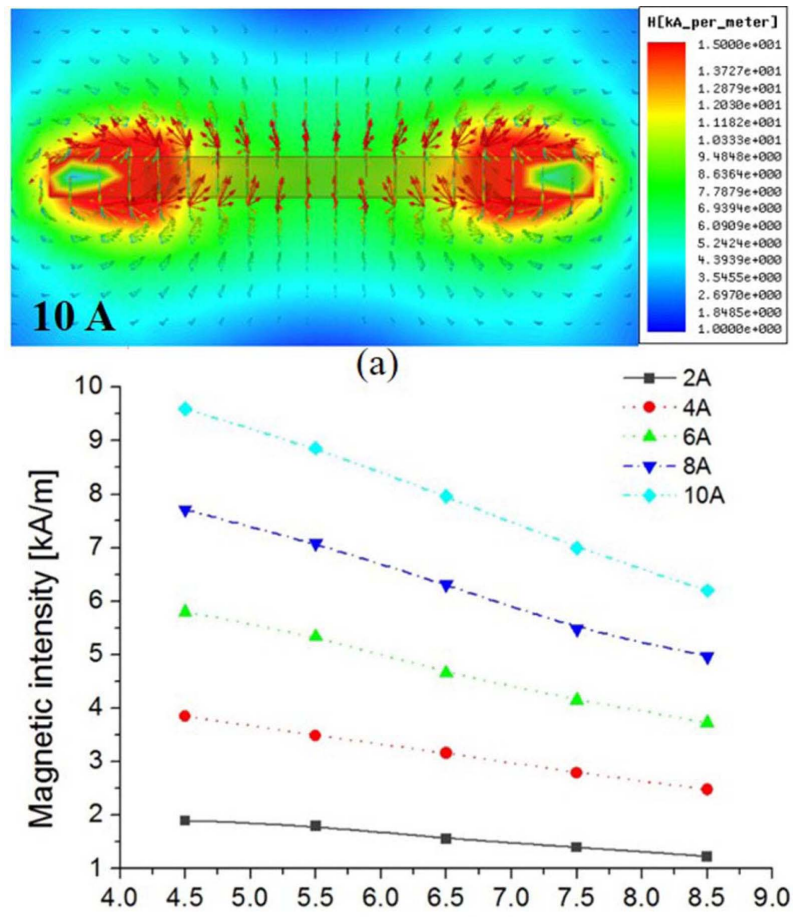

(b) Height [cm]

Fig. 3. (Color online) (a) Magnetic field simulation of the driving coil at input current of $10 \mathrm{~A}$ and (b) measurement results of magnetic field strength on the driving coil according to changes in height and input current.
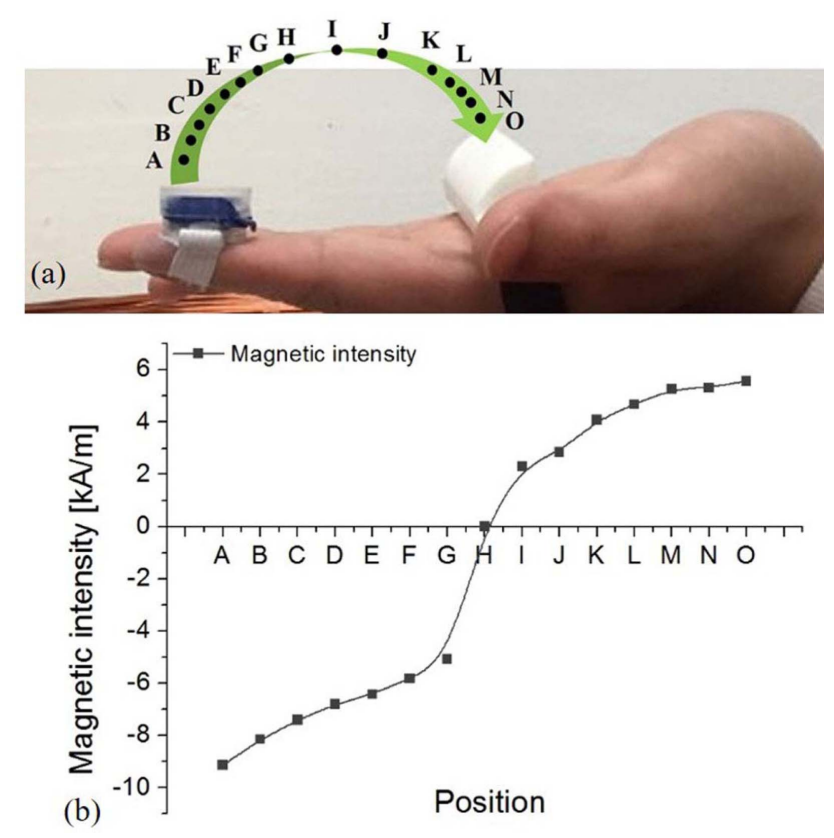

Fig. 4. (Color online) (a) Trajectory of forefinger and (b) magnetic field strength according to the trajectory.

flexion motion, a magnetic field strength of $2.3 \mathrm{kA} / \mathrm{m}$ was required, whereas the extension motion required a minimum

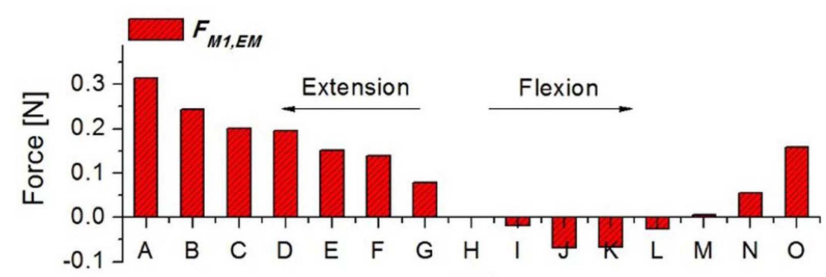

(a)
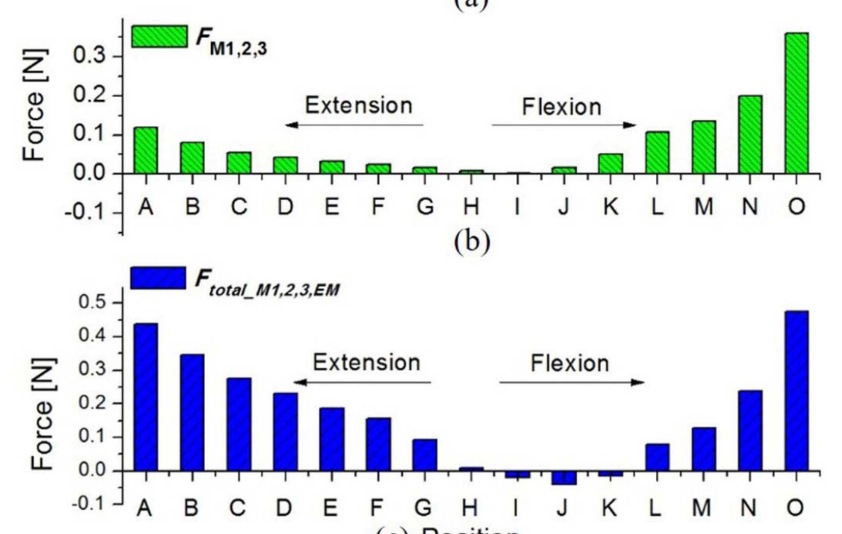

(c) Position

Fig. 5. (Color online) Magnetic force analysis for extension and flexion motions: (a) force variations by $\boldsymbol{F}_{\boldsymbol{M I}, \boldsymbol{E M}}$, (b) mag-

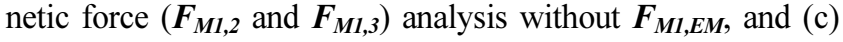
total force analysis of extension and flexion motions. A to $\mathrm{O}$ denote positions of finger.

of magnetic field strength of $-5.08 \mathrm{kA} / \mathrm{m}$. Because of musculoskeletal characteristics, extension motion requires a higher force than flexion motion. When the forefinger reached the maximum extension point $\mathrm{A}$ and the flexion point $\mathrm{O}$, the magnetic field strengths were $-9.13 \mathrm{kA} / \mathrm{m}$ and $5.56 \mathrm{kA} / \mathrm{m}$, respectively.

We conducted a magnetic force analysis, as shown in Fig. 5. In the results, a plus symbol on the forces denotes an attractive force, whereas a minus symbol means a repulsive force. For the force analysis, we considered all magnetic forces in this system $\left(\boldsymbol{F}_{\boldsymbol{M 1 , 2}}, \boldsymbol{F}_{\boldsymbol{M 1}, 3}\right.$, and $\left.\boldsymbol{F}_{\boldsymbol{M 1 , E M}}\right)$. The total force is expressed by the sum of all magnetic forces. For extension motion, $\boldsymbol{F}_{\boldsymbol{M I}, \boldsymbol{E M}}$ and $\boldsymbol{F}_{\boldsymbol{M 1 , 3}}$ become attractive forces in the proposed system. Therefore, points from $\mathrm{H}$ (the initial point) to A showed extension motion. Point $\mathrm{A}$ is the maximum extension motion. In this case, the applied magnetic field was $-9.13 \mathrm{kA} / \mathrm{m}$, and $\boldsymbol{F}_{\boldsymbol{M 1}, \boldsymbol{E M}}$ was $0.315 \mathrm{~N}$. To reach a maximum, the magnetic field and $\boldsymbol{F}_{\boldsymbol{M 1 , E M}}$ gradually increased. In the case of flexion motion, $\boldsymbol{F}_{\boldsymbol{M I} \text {, EM }}$ represented two forces, both an attractive force and a repulsive force, as shown in Fig. 5(a). The repulsive force of $\boldsymbol{F}_{\boldsymbol{M I}, \boldsymbol{E M}}$ pushed M1 and caused a rotation of the finger joint. When $\mathrm{M} 1$ reached point $\mathrm{M}$, the $\mathrm{S}$ pole on $\mathrm{M} 1$ and the $\mathrm{N}$ pole on the electromagnet (driving coil) were face-to-face. Thus, the points from $\mathrm{M}$ to $\mathrm{O}$ gene- 
rated an attractive force. The maximum of $\boldsymbol{F}_{M 1, E M}$ was $0.159 \mathrm{~N}$ in the flexion motion at point O. Figure 5(b) shows the magnetic force between magnets $\left(\boldsymbol{F}_{M 1,2}\right.$ and $\boldsymbol{F}_{\boldsymbol{M} 1,3)}$. The points from $\mathrm{H}$ to $\mathrm{A}$ are influenced by the attractive force of $\boldsymbol{F}_{\boldsymbol{M} 1,3}$ between M1 and M3 during extension motion. The points from I to $\mathrm{O}$ show the attractive force of $\boldsymbol{F}_{\boldsymbol{M} 1,2}$ during flexion motion. Because the distance between M1 and M3 is longer than that between M1 and M2. $\boldsymbol{F}_{\boldsymbol{M} \mathbf{1}, \mathbf{2}}$ is higher than $\boldsymbol{F}_{\boldsymbol{M} \mathbf{1}, \mathbf{3}}$. At point A, the distance between M1 and M3 is $4.5 \mathrm{~cm}$, whereas the distance between $\mathrm{M} 1$ and $\mathrm{M} 2$ is $1.9 \mathrm{~cm}$ at point $\mathrm{O}$. The maximums of $\boldsymbol{F}_{\boldsymbol{M} 1,3}$ and $\boldsymbol{F}_{\boldsymbol{M} 1,2}$ were $0.119 \mathrm{~N}$ and $0.36 \mathrm{~N}$, respectively. $\boldsymbol{F}_{\boldsymbol{M I , 3}}$ and $\boldsymbol{F}_{\boldsymbol{M I , 2}}$ enhanced the extension motion and flexion motion based on the two motions by $\boldsymbol{F}_{\boldsymbol{M I}, \boldsymbol{E M}}$. Figure 5(c) shows the total force for the two motions. In the flexion motion, the total points of repulsive force decreased from I to $\mathrm{K}$ because of the sum of the force between $\boldsymbol{F}_{\boldsymbol{M I , 2}}$ and $\boldsymbol{F}_{M 1, E M}$. The starting force and maximum force were 0.094 $\mathrm{N}$ and $0.438 \mathrm{~N}$ at points $\mathrm{G}$ and $\mathrm{A}$, respectively. During flexion motion, the starting force was $-0.019 \mathrm{~N}$ at point $\mathrm{I}$, and the maximum flexion was $0.474 \mathrm{~N}$. As mentioned before, in general, the generation of extension motion is difficult to compare flexion motion of stroke patients. Thus, the extension motion requires a higher threshold force than that of flexion motion. When we compared forces from $\mathrm{E}$ to $\mathrm{G}$ (extension) and from I to K (flexion),

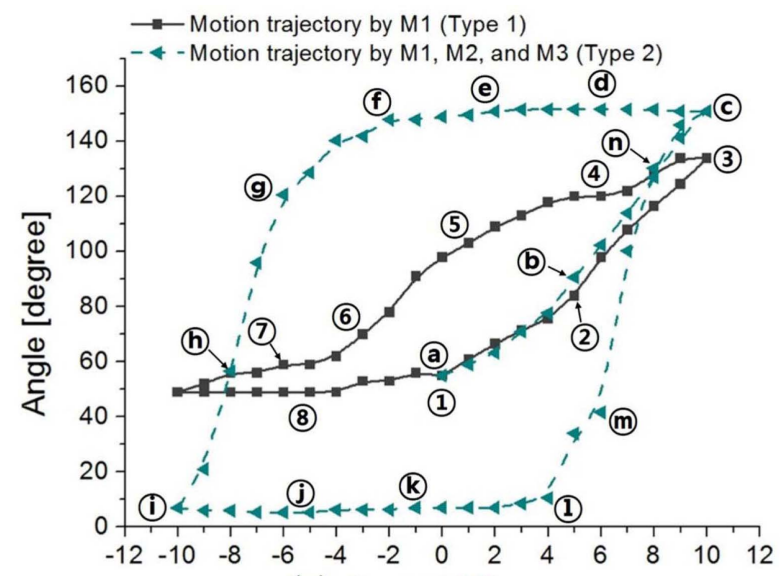

(a) Current $[A]$

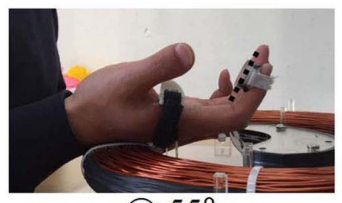

(a) $55^{\circ}$

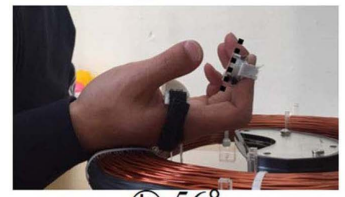

(h) $56^{\circ}$

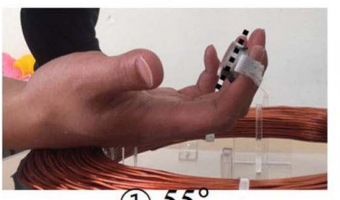

(1) $55^{\circ}$

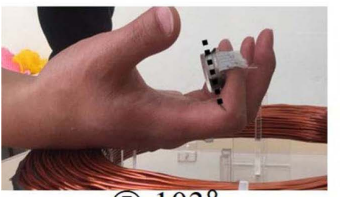

(5) $103^{\circ}$



(b) $90^{\circ}$

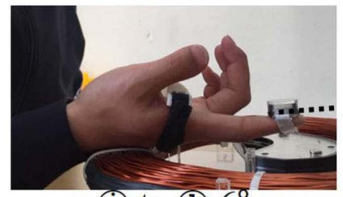

(i) to (1) $6^{\circ}$

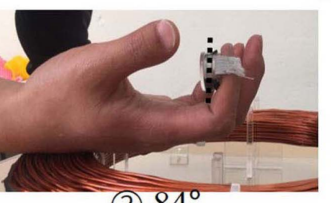

(2) $84^{\circ}$



(6) $70^{\circ}$

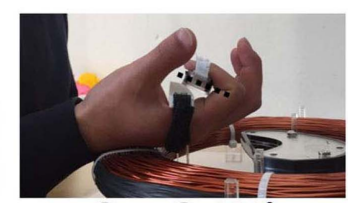

(c) to $f 151^{\circ}$

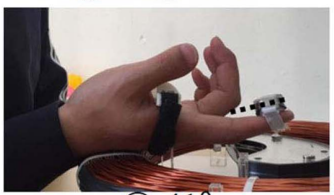

(b)

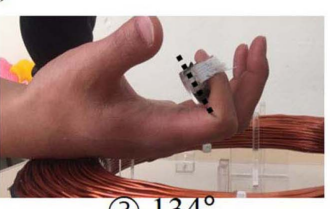

(3) $134^{\circ}$

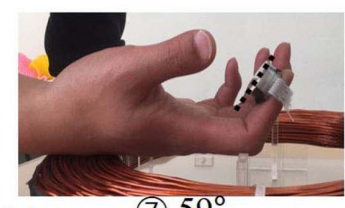

(c)

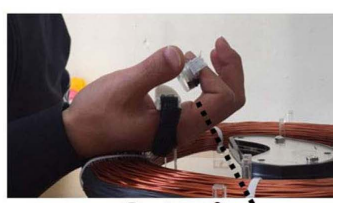

(g) $120^{\circ}:$

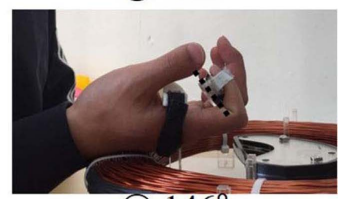

(n) $146^{\circ}$

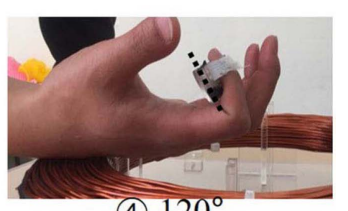

(4) $120^{\circ}$

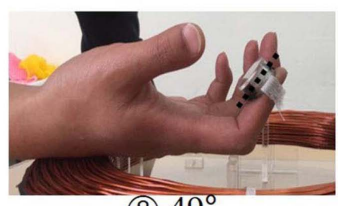

(8) $49^{\circ}$

Fig. 6. (Color online) (a) Hysteresis characteristics of extension and flexion motions in the system and (b) motion captures according to variations in finger angle and the driving current. 
the extension force was, on average, six times higher than the flexion force.

Finally, we performed motion analysis according to changes in the applied current signal. In particular, we compared the influence of $\boldsymbol{F}_{\boldsymbol{M 1 , 3},}$ and $\boldsymbol{F}_{\boldsymbol{M} 1,2}$ on finger motions. Figure 6(a) shows variations in finger movement according to changes in the input current signals. The dashed line indicates the results, including the impact on $\boldsymbol{F}_{\boldsymbol{M 1 , 3},}$ and $\boldsymbol{F}_{\boldsymbol{M 1 , 2}}$, and a solid line shows the results of $\boldsymbol{F}_{\boldsymbol{M I}, \boldsymbol{E M}}$ without M2 and M3. The driving current was controlled by three conditions: 0 to $10 \mathrm{~A}, 10$ to $-10 \mathrm{~A}$, and -10 to $10 \mathrm{~A}$. Under these conditions, the finger motion produced hysteresis movements in the system. Because type 1 utilized only $\boldsymbol{F}_{\boldsymbol{M I}, E M}$, the driving force was weaker than that of type 2 . Therefore, the variations in the finger's angle were lower than those of type 2 . In addition, the magnetic force of $\boldsymbol{F}_{\boldsymbol{M I , 3}}$ and $\boldsymbol{F}_{\boldsymbol{M I , 2}}$ in type 2 maintained maximum extension and flexion motions. In particular, the maintained extension motion (i) to (1) provided a stretching effect.

In type 1 , the maximum flexion angle was $134^{\circ}$ (the angular variation was $79^{\circ}$ ), and the minimum extension angle was $49^{\circ}$ (the angular variation was $6^{\circ}$ ), as shown Fig. 6(c), whereas type 2 generated a maximum flexion angle of $151^{\circ}$ (the angular variation was $96^{\circ}$ ), and the minimum extension angle was $6^{\circ}$ (with an angular variation of $49^{\circ}$ ), as shown in Fig. 6(b). These differences between type 1 and type 2 resulted from $\boldsymbol{F}_{\boldsymbol{M I , 3}}$ and $\boldsymbol{F}_{\boldsymbol{M I , 2}}$. In particular, $\boldsymbol{F}_{\boldsymbol{M} 1,3}$ significantly improved the extension motion. Figure 6(b) shows the finger movements in type 2.

The points from (a) to (c) denote the flexion motion $\left(F_{\text {total }}=F_{M 1, \mathrm{EM}}+F_{M 1,2}\right)$, (C) to (f) show the maintained maximum flexion $\left(F_{M 1,2}>F_{M 1, \mathrm{EM}}+F_{\text {react }}\right)$, where $F_{\text {react }}$ denotes a reaction force of the tendon, (f) to (i) show the motion conversion from flexion to extension motions where the conditions are divided into $F_{M 1,2} \leq F_{M 1, \mathrm{EM}}+$ $F_{\text {react }}\left(55^{\circ} \leq \theta \leq 151^{\circ}\right)$ and $F_{M 1,2} \leq F_{M 1, \mathrm{EM}}+F_{\text {react }}+$ $F_{M 1,3}\left(6^{\circ} \leq \theta \leq 55^{\circ}\right)$, (i) to (1) show the maintained extension motion, where $F_{M 1,3} \gg F_{M 1, \mathrm{EM}}+F_{\text {react }}$, and (1) to (c) show rapidly converted motion from extension to flexion motion, where driving conditions are divided into $F_{M 1,3} \leq F_{M 1, \mathrm{EM}}+F_{\text {react }} \quad\left(6^{\circ} \leq \theta \leq 55^{\circ}\right) \quad$ and $F_{M 1,3} \leq F_{M 1, \mathrm{EM}}+F_{M 1,2}+F_{\text {react }}\left(55^{\circ} \leq \theta \leq 151^{\circ}\right)$.

The reaction force from the tendon acted in the total force, but we ignored the reaction force in the total force analysis. As mentioned above, the method can produce twist motion of the finger by the position between M1 and the driving coil. Figure 7 shows twist motion of the finger. The direction of the magnetic moment of M1 is perpendicular to the driving coil at the reference angle of $0^{\circ}$. The driving currents of $10 \mathrm{~A}$ and $-10 \mathrm{~A}$ caused the twist angles of $-32^{\circ}$ and $26^{\circ}$, respectively.

\section{Discussion and Conclusion}

In this study, we proposed a nonmechanical hand rehabilitation method using magnetic force. Most hand rehabilitation mechanisms depend on mechanical system, such as robotic hands. Because of the mechanical components, their configuration is very complex, and installation on the hand is difficult. The proposed method simply installed a magnet on the finger without using mechanical components, and we controlled the magnetic field strength and the direction of the driving coil for extension and flexion motions. In the system, the magnetic force $\boldsymbol{F}_{\boldsymbol{M} \mathbf{E}, E M}$ between M1 and the driving coil generated a weak extension motion. In finger rehabilitation, extension motion is important and it is not easy to generate such motion for stroke patients. To solve this problem, we installed magnet M3 in the driving coil. As a result, the additional force $\boldsymbol{F}_{\boldsymbol{M} 1,3}$ improved the extension force to a maximum of 9.8 times the force. In addition, the magnet M2 enhanced flexion motion by $\boldsymbol{F}_{\boldsymbol{M} 1,2}$. Furthermore, we verified twist motion of the finger by the changed orientation of the hand. Through various experiments and analyses, we verified the active actuation of the finger by magnetic interaction for finger rehabilitation. Although our experiment was conducted on a normal human, we were able to
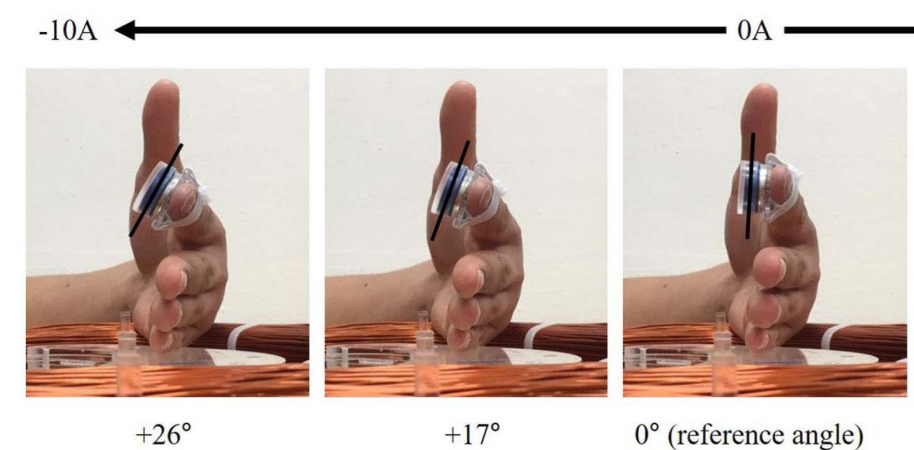

$0^{\circ}$ (reference angle)

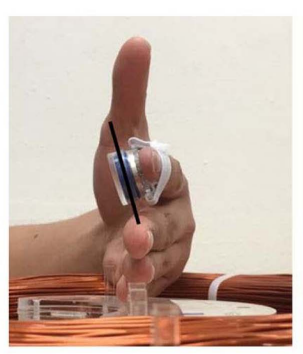

$-19^{\circ}$ $+10 \mathrm{~A}$

Fig. 7. (Color online) Twist motions of a finger according to changes in driving currents. 
observe the external force and active actuations for finger rehabilitation. In the near future, we will confirm the effects of the proposed method through clinical application. In particular, we will consider increase of DOFs for the finger motion. Because of the limited hand motion of stroke patients, the position of the hand is limited at a single coil. Therefore, to improve DOFs of finger motion, three axis coil system is required. The three axis coil system can cause finger movement in all directions.

\section{Acknowledgments}

This research was supported by Wonkwang University in 2017 .

\section{References}

[1] E. B. Brokaw, I. Black, R. J. Holley, and P. S. Lum. IEEE Trans. Neural Syst. 19, 391 (2011).

[2] A. Chiri, N. Vitiello, F. Giovacchini, and S. Roccella, IEEE/ASME Trans. Mechatronics. 17, 884 (2012).

[3] M. Bouzit, G. Burdea, G. Popescu, and R. Boian, IEEE/ ASME Trans. Mechatronics. 7, 256 (2002).

[4] W. H. Chang and Y. H. Kim, Journal of Stroke. 15, 174 (2013).
[5] M. Pignolo, J. Rehabil Med. 41, 955 (2009).

[6] S. Biggar and W. Yao, IEEE Trans. Neural. Syst. Rehabil. Eng. 24, 1071 (2016).

[7] D. Laonardis, M. Barsotti, C Loconsole, M. Solazzi, M. Troncossi, C. Mazzotti, V. P. Castelli, C. Procopio, G. Lamola, C. Chisari, M. Bergamasco, and A. Frisoli, IEEE Trans. Haptics. 8, 140 (2015).

[8] H. C. Fischer, K. M. Triandafilou, K. O. Thielbar, J. M. Ochoa, E. D. Lazzaro, K. A. Pacholski, and D. G. Kamper, IEEE Trans. Neural Syst Rehabil. Eng. 24, 344 (2016).

[9] G. Belforte, G. Eula, A. Ivanov, and S. Sirolli, Actutors. 3, 84 (2014).

[10] H. C. Fischer, K. Stubblefield, T. Kline, X. Luo, R. V. Kenyon, and D. G. Kamper, Topics Stroke Rehabil. 12, 1 (2007).

[11] C. H. Yu and S. H. Kim, J. Magn. 21, 616 (2016).

[12] S. H. Kim, S. Hashi, and K. Ishiyama, IEEE Trans. Mag. 49, 3488 (2013).

[13] S. H. Kim and K. Ishiyama, IEEE/ASME Trans. Mechatronics. 19, 1651 (2014).

[14] E. P. Furlani, Permanent Magnet and Electromechanical Devices, Academic Press, San Diego (2001) pp. 126-142.

[15] D. Vokoun, M. Beleggia, L. Heller, and P. Sittner, JMMM. 321, 3758 (2009).

[16] L. Kocbach and S. Lubbad, Phys. Educ. 45, 345 (2010). 